\title{
Discovery of spectral variation in the optical counterpart of IRAS $01005+7910$
}

\author{
V. G. Klochkova ${ }^{1,2}$, M. V. Yushkin ${ }^{1,2}$, A. S. Miroshnichenko ${ }^{3,4}$, V. E. Panchuk ${ }^{1,2}$, and K. S. Bjorkman ${ }^{3}$ \\ 1 Special Astrophysical Observatory of the Russian Academy of Sciences, Karachai-Cirkassian Republic, \\ Nizhnij Arkhyz 369167, Russia \\ ${ }^{2}$ Isaac Newton Institute of Chile, SAO Branch \\ 3 Ritter Observatory, Dept. of Physics \& Astronomy, University of Toledo, Toledo, OH 43606-3390, USA \\ 4 Central Astronomical Observatory of the Russian Academy of Sciences at Pulkovo, 196140 Saint-Petersburg, Russia \\ Received 1 March 2002 / Accepted 29 May 2002
}

\begin{abstract}
We present a study of the high-resolution spectroscopic data for the proto-planetary nebula candidate IRAS $01005+7910$. For the first time a careful spectral line identification is carried out, and a significant variability of the optical spectrum is detected. We found absorption lines of $\mathrm{C}_{\text {IIIIII, }} \mathrm{N}_{\text {II, }} \mathrm{O}_{\text {II }}, \mathrm{Al}_{\text {III }}, \mathrm{Si}$ III, and $\mathrm{Mg}$ II $(\lambda 4481 \AA$ A), as well as emission lines of $\mathrm{Si}$ II and [Fe II]. Both absorption and emission components are present in the Balmer lines, Na I resonance $\mathrm{D}_{1,2}$ lines, $\mathrm{He}_{\mathrm{I}}$, and Fe III lines. The He I line profiles vary from straight to inverse P Cyg-type on a timescale of days to months. The resonance $\mathrm{Na}$ I lines show 5 absorption components at a resolution of $R=60000$. Additionally, the $\mathrm{Na}_{\mathrm{I}} \mathrm{D}_{2}$ line exhibits a variable emission component with a width comparable to that of the Balmer line emission components. Using the model atmospheres method within the LTE-approximation, the effective temperature $\left(T_{\text {eff }} \sim 21500 \mathrm{~K}\right)$, the metallicity $[\mathrm{Fe} / \mathrm{H}]_{\odot}=-0.31$, and the ratio $\mathrm{C} / \mathrm{O}>1$ is reported. Finally, we suggest that IRAS $01005+7910$ is a carbon-rich post-AGB star with a luminosity $\log \left(L / L_{\odot}\right)=3.6$ at a distance about $3 \mathrm{kpc}$.
\end{abstract}

Key words. stars: evolution - stars: AGB and post-AGB - stars: emission-line - stars: individual: IRAS 01005+7910 techniques: spectroscopic

\section{Introduction}

Proto-planetary nebulae (PPNe) are post-AGB objects evolving toward the Planetary Nebula (PN) stage with increasing effective temperatures $\left(T_{\text {eff }}\right)$ and almost constant luminosities (e.g. Blöcker 1995). They span a range of spectral types between $B$ and $G$ and are surrounded by a significant amount of circumstellar dust, thanks to which many such sources were detected by the IRAS satellite (Parthasarathy \& Pottasch 1989). Follow-up observations showed that PPNe, especially those of early spectral types, exhibit variable emission-line spectra (Parthasarathy et al. 2000). Spectroscopic studies are crucial for constraining PPNe masses through determination of the fundamental parameters and their evolutionary state with measurements of the chemical composition and mass loss rate. Spectroscopic monitoring programmes, which are still rare, are important for studies of physical processes in the objects' immediate vicinity and for detecting binary PPNe (Van Winckel et al. 1995), whose population recently began to grow as a result of such programmes (see Van Winckel 2001 for a review).

Here we present the first results obtained in the course of spectroscopic monitoring of a poorly-studied PPN candidate IRAS 01005+7910 (hereafter IRAS 01005) at the

Send offprint requests to: V. G. Klochkova,

e-mail: valenta@sao.ru 6-m telescope of the Russian Academy of Sciences. The object is located far from the Galactic plane $\left(b=16^{\circ}\right)$ and is identified with an 11-mag. peculiar star. Its IRAS colours are similar to those of PPNe, lying in the region $v$ in the IRAS two-colour diagram of Van der Veen \& Habing (1988). However, in contrast to most of the PPNe, maser emission from IRAS 01005 has been detected neither in ${ }^{12} \mathrm{CO}$ nor in OH bands (Likkel 1989; Likkel et al. 1991; Omont et al. 1993). According to a chronological sequence suggested by Lewis (1989), this result indicates that the object is very close to the PN stage. The IR spectrum of IRAS 01005 (Hrivnak et al. 2000) contains emission bands at $3.3,6.2,7.7,8.6,11.3,26$, and $30 \mu \mathrm{m}$ which are characteristic of carbon-rich PPNe. At the same time, IRAS 01005 does not show the famous emission at $21 \mu \mathrm{m}$, whose presence is attributed to an excess of the s-process elements (Klochkova 1998; Decin et al. 1998).

So far only low-resolution spectra of IRAS 01005 have been published by $\mathrm{Hu}$ (2001), who classified the object as B2 re. This author also mentioned no changes in a P Cyg-type $\mathrm{H} \alpha$ line profile in his spectra, obtained 10 years apart. No photometric variations of the object have been reported.

\section{Observations and data reduction}

The observations were obtained at the 6-m telescope of the Special Astrophysical Observatory (SAO) of the Russian 
Table 1. Observing log for IRAS $01005+7910$.

\begin{tabular}{ccccc}
\hline \hline Date & $\begin{array}{c}\text { Exposure } \\
\text { time, } \mathrm{s}\end{array}$ & $\begin{array}{c}\text { Sp.range } \\
\AA\end{array}$ & $\begin{array}{c}\text { Resolv. } \\
\text { power, } R\end{array}$ & $\overline{\overline{S / N}}$ \\
\hline $06 / 07 / 2000$ & $2 \times 3200$ & $4300-7800$ & 15000 & 100 \\
$13 / 07 / 2000$ & $2 \times 3200$ & $4300-7800$ & 15000 & 180 \\
$09 / 11 / 2000$ & $2 \times 3200$ & $4720-6320$ & 30000 & 120 \\
$03 / 01 / 2001$ & $2 \times 2400$ & $4950-6600$ & 15000 & 220 \\
$11 / 08 / 2001$ & $2 \times 3200$ & $5300-6680$ & 30000 & 100 \\
$02 / 12 / 2001$ & $2 \times 3600$ & $4600-6070$ & 60000 & 75 \\
$25 / 01 / 2002$ & $2 \times 3600$ & $3500-5070$ & 60000 & 40 \\
$04 / 02 / 2002$ & $2 \times 3600$ & $4600-6070$ & 60000 & 55 \\
\hline
\end{tabular}

Academy of Sciences with the échelle-spectrograph PFES (mounted at the prime focus, $1 \mathrm{~K} \times 1 \mathrm{~K}$ CCD-chip, resolving power $R=15000$, Panchuk et al. 1998), the multimode échelle-spectrograph LYNX (Nasmyth-2 focus, $1 \mathrm{~K} \times 1 \mathrm{~K}$ CCD-chip, $R=30000$, Panchuk et al. 1999a), and the échelle-spectrograph NES (Nasmyth-2 focus, $2 \mathrm{~K} \times 2 \mathrm{~K}$ CCDchip, $R=60000$, Panchuk et al. 1999b). The observing log is presented in Table 1 . The cosmic ray traces were removed by median averaging of two subsequent spectra. A hollow cathode Th-Ar lamp was used for the wavelength calibration.

First steps of the data reduction process (cosmic ray trace removal, background subtraction, and spectral order extraction) were done under the ECHELLE context of MIDAS (version 01FEB), while the final steps (normalization to the continuum level and radial velocity $\left(V_{\mathrm{r}}\right)$ and equivalent width measurements) were completed using a package DECH20 (Galazutdinov 1992).

\section{Main results}

\subsection{The spectrum of IRAS $01005+7910$}

The optical spectrum of IRAS 01005 is a combination of the photospheric spectrum of a hot star and circumstellar emission lines. The line identification was completed using several line lists of Kilian \& Nissen (1989), Parthasarathy et al. (2000), a multiplet table of Moore (1945) and our own experince in identification of spectra of related objects IRAS $18062+2410$ (Arkhipova et al. 2001a) and V1853 Cyg (Arkhipova et al. 2001b). Refined line wavelengths, oscillator strengths and excitation potentials were taken from the data base VALD (Piskunov et al. 1995). Absorption lines of $\mathrm{C}_{\text {II }} / \mathrm{III}, \mathrm{N}_{\text {II, }}$ O II, Al III, Si III, and the Mg II line at $\lambda 4481 \AA$ are present in the spectrum of IRAS 01005. Pure emission features were identified with Si II lines and forbidden lines of [Fe II]. Both emission and absorption components are detected in the hydrogen Balmer lines, the resonance lines of the $\mathrm{Na}_{\mathrm{I}} \mathrm{D}_{1,2}$ doublet, He I lines, and Fe III lines. We also found the Si II doublet $\lambda \lambda 4128,4131 \AA$ in absorption with the equivalent widths of 60 and $70 \mathrm{~m} \AA$, respectively. The absence of lines with low excitation potentials and those of neutral elements (except for those of $\mathrm{H}_{\mathrm{I}}, \mathrm{He}_{\mathrm{I}}$, and $\mathrm{NaI}$ ) indicates that the star has a high $T_{\text {eff. The full list }}$ of identified emission and absorption lines in the spectrum of IRAS 01005 in the region 4300-7800 $\AA$ is presented in Table 2. Unidentified lines are denoted by "UN".

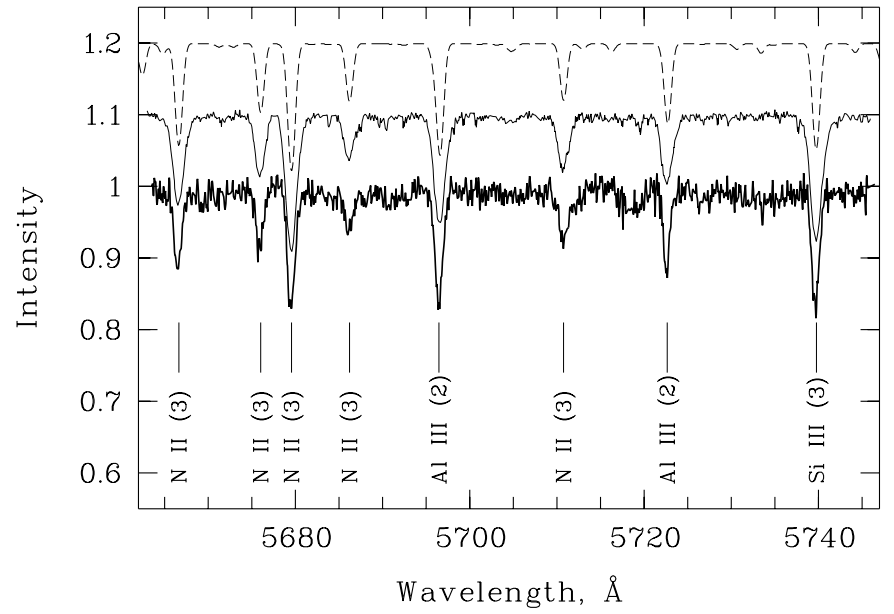

Fig. 1. Nitrogen lines in the spectrum of IRAS $01005+7910$ (thick line). The dashed line represents a synthetic spectrum for $T_{\text {eff }}=$ $21000 \mathrm{~K}, \log g=3.0$ and $\xi_{\mathrm{t}}=15 \mathrm{~km} \mathrm{~s}^{-1}$. The spectrum of 9 Cep (thin line) is plotted between the theoretical one and that of IRAS 01005 .

Our spectra of IRAS 01005 contain several diffuse interstellar bands. The most intense of them are the following: $\lambda 5780 \AA\left(W_{\lambda}=100 \mathrm{~m} \AA\right), \lambda 5797 \AA\left(W_{\lambda}=40 \mathrm{~m} \AA\right)$ and $\lambda 6613 \AA$ $\left(W_{\lambda}=30 \mathrm{~m} \AA\right)$. Their $V_{\mathrm{r}} \sim-10 \mathrm{~km} \mathrm{~s}^{-1}$ agree with the interstellar origin. The strength of the $\lambda 5780 \AA$ band corresponds to the interstellar reddening $E_{B-V}=0.2$ or $A_{V}=0.6$ (Herbig 1993).

\subsection{Spectral type and abundance calculation}

In order to estimate the spectral type of IRAS 01005 we used a calibration for supergiants based on the line equivalent widths from Didelon (1982). Using only the lines without noticeable emission components ( $\mathrm{Mg}_{\text {II }} \lambda 4481 \AA$, Si III $\lambda \lambda 4553,4575 \AA$ and Si II $\lambda 4128 \AA$ ), we derived an average type of B1.7 \pm 0.5 which is in agreement with the abovementioned result of $\mathrm{Hu}$ (2001). We also compared the spectrum of IRAS 01005 with that of the normal supergiant $9 \mathrm{Cep}$ (B2 Ib) and found them very similar (Fig. 1). While the strength of the nitrogen and oxygen lines in the spectrum of IRAS 01005 is in agreement with the estimated spectral type, the carbon lines are somewhat stronger (Fig. 2) suggesting its overabundance. The latter agrees with the object's carbon-rich IR spectrum.

We note here that these estimates are based on the spectral type criteria for population I stars. Application of these criteria to stars with a possible metal deficiency and evolutionarilyaltered abundances of light elements can insert significant systematic errors in the spectral classification. Nevertheless, the absence of He II and Si IV lines indicates that $T_{\text {eff }} \leq 25000 \mathrm{~K}$, while the presence of He I lines in emission places a lower limit of $T_{\text {eff }}$ at $\sim 18000-20000 \mathrm{~K}$ (e.g., Miroshnichenko et al. 1998).

For an independent $T_{\text {eff }}$ estimate, we compared the observed spectrum with a synthetic one, calculated from the Kurucz (1993) LTE models with the solar chemical composition. The best fit was obtained for $T_{\mathrm{eff}}=21000 \mathrm{~K}$, microturbulent velocity $\xi_{\mathrm{t}}=15 \mathrm{~km} \mathrm{~s}^{-1}$, and surface gravity $\log g=3.0$ (see Figs. 1 and 2). 
Table 2. Emission (e) and absorption (a) lines indentified in the specrtum of IRAS 01005+7910. $V_{\mathrm{r}}^{1}$ and $V_{\mathrm{r}}^{2}$ are the heliocentric $V_{\mathrm{r}}$ at two epochs (July 13, 2000 and December 2, 2001 respectively). $W_{\lambda}$ is the equivalent width for the lines in the spectrum of July 13, 2000.

\begin{tabular}{|c|c|c|c|c|c|c|c|c|c|c|c|c|c|}
\hline $\begin{array}{l}\lambda_{\text {lab }} \\
\AA \\
\end{array}$ & Spec. (mult) & $\log (g f)$ & & $\begin{array}{l}W_{\lambda} \\
\AA \\
\end{array}$ & $\begin{array}{c}V_{\mathrm{r}}^{1} \\
\mathrm{~km} \mathrm{~s}^{-1} \\
\end{array}$ & $\begin{array}{c}V_{\mathrm{r}}^{2} \\
\mathrm{~km} \mathrm{~s}^{-1}\end{array}$ & $\begin{array}{c}\lambda_{\text {lab }} \\
\AA \\
\end{array}$ & Spec. (mult) & $\log (g f)$ & & $\begin{array}{l}W_{\lambda} \\
\AA \\
\end{array}$ & $\begin{array}{c}V_{\mathrm{r}}^{1} \\
\mathrm{~km} \mathrm{~s}^{-1} \\
\end{array}$ & $\begin{array}{c}V_{\mathrm{r}}^{2} \\
\mathrm{~km} \mathrm{~s}^{-1} \\
\end{array}$ \\
\hline 4317.136 & O II (2) & -0.386 & $\mathrm{a}$ & 0.107 & -39 & & 5073.903 & Fe III (5) & -2.557 & $\mathrm{a}$ & 0.034 & -40 & \\
\hline 4319.625 & O II (2) & -0.380 & a & 0.145 & -46 & & 5086.701 & Fe III (5) & -2.590 & a & 0.032 & -21 & \\
\hline 4325.764 & O II (2) & -1.099 & $\mathrm{a}$ & & blend & & 5127.463 & Fe III (5) & -2.057 & $\mathrm{e}$ & 0.017 & -72 & -68 \\
\hline 4325.993 & C II (28) & -0.089 & $\mathrm{a}$ & & blend & & & & & $\mathrm{a}$ & 0.027 & -27 & -23 \\
\hline 4331.813 & O II (41) & -0.090 & $\mathrm{a}$ & 0.027 & -39 & & 5133.121 & C II (16) & 0.107 & $\mathrm{a}$ & 0.062 & -46 & -27 \\
\hline 4336.861 & O II (2) & -0.762 & $\mathrm{a}$ & 0.078 & -42 & & 5143.497 & C II (16) & -0.212 & $\mathrm{a}$ & 0.050 & -49 & \\
\hline 4340.462 & H I (1) & -0.447 & $\mathrm{e}$ & 0.205 & -43 & & 5145.167 & C II (16) & 0.189 & $\mathrm{a}$ & 0.098 & -54 & -31 \\
\hline 4345.558 & O II (2) & -0.346 & $\mathrm{a}$ & 0.105 & -50 & & 5151.085 & C II (16) & -0.179 & $\mathrm{a}$ & 0.038 & -46 & -36 \\
\hline 4347.379 & O II (16) & 0.029 & $\mathrm{a}$ & 0.045 & -48 & & 5156.111 & Fe III (5) & -2.018 & $\mathrm{e}$ & 0.025 & -72 & -66 \\
\hline 4349.427 & O II (2) & 0.060 & a & 0.178 & -49 & & & & & a & 0.029 & -28 & -22 \\
\hline 4351.268 & O II (16) & 0.251 & $\mathrm{a}$ & 0.060 & -43 & & 5158.810 & {$[\mathrm{FeII}](19 \mathrm{~F})$} & & $\mathrm{e}$ & 0.040 & -58 & -51 \\
\hline 4359.340 & [FeII] (7F) & & $\mathrm{e}$ & 0.040 & -56 & & 5197.577 & Fe II (49) & -2.100 & e & 0.067 & -32 & -32 \\
\hline 4366.893 & O II (2) & -0.348 & $\mathrm{a}$ & 0.140 & -53 & & 5199 & UN & & $\mathrm{e}$ & 0.005 & & \\
\hline 4387.929 & $\mathrm{He} \mathrm{I} \mathrm{(51)}$ & -0.883 & $\mathrm{a}$ & 0.390 & -41 & & 5200 & UN & & $\mathrm{e}$ & 0.040 & & \\
\hline 4411.364 & C II (39) & 0.918 & $\mathrm{a}$ & 0.036 & -60 & & 5206.650 & O II (32) & -0.266 & a & 0.021 & -35 & \\
\hline 4414.900 & O II (5) & 0.172 & $\mathrm{a}$ & 0.180 & -53 & & 5219 & UN & & a & 0.022 & & \\
\hline 4416.980 & O II (5) & -0.077 & $\mathrm{a}$ & 0.120 & -46 & & 5227.830 & $\mathrm{Fe}$ III & -0.055 & a & 0.017 & -45 & \\
\hline 4437.551 & $\mathrm{He} \mathrm{I}(50)$ & -2.034 & $\mathrm{a}$ & 0.094 & -41 & & 5261.620 & {$[\mathrm{FeII}](19 \mathrm{~F})$} & & $\mathrm{e}$ & 0.015 & -59 & \\
\hline 4447.030 & N II (55) & 0.285 & $\mathrm{a}$ & 0.088 & -49 & & 5273.380 & {$[\mathrm{FeII}](18 \mathrm{~F})$} & & $\mathrm{e}$ & 0.010 & -62 & -52 \\
\hline 4452.380 & O II (5) & -0.789 & $\mathrm{a}$ & 0.044 & -41 & & 5357 & UN & & $\mathrm{e}$ & 0.065 & & \\
\hline 4471.485 & $\mathrm{He} \mathrm{I} \mathrm{(14)}$ & 0.053 & a & 0.430 & -26 & & 5454.214 & N II (29) & -0.827 & a & 0.042 & -48 & \\
\hline 4481.207 & Mg II(4) & 0.985 & $\mathrm{a}$ & 0.190 & -33 & & 5463 & UN & & a & 0.035 & & \\
\hline 4512.565 & Al III (3) & 0.410 & $\mathrm{a}$ & 0.026 & -41 & & 5495.653 & N II (29) & -0.266 & $\mathrm{a}$ & 0.036 & -47 & \\
\hline 4529.164 & Al III (3) & 0.706 & $\mathrm{a}$ & 0.072 & -47 & & 5639 & UN & & $\mathrm{a}$ & 0.050 & & \\
\hline 4552.622 & Si III (2) & 0.181 & $\mathrm{a}$ & 0.274 & -51 & & 5646 & UN & & $\mathrm{a}$ & 0.035 & & \\
\hline 4567.840 & Si III (2) & -0.039 & $\mathrm{a}$ & 0.220 & -53 & -29 & 5662.459 & C II (15) & -0.249 & a & 0.025 & -44 & \\
\hline 4574.757 & Si III (2) & -0.509 & $\mathrm{a}$ & 0.110 & -51 & & 5666.627 & N II (3) & -0.045 & $\mathrm{a}$ & 0.118 & -42 & -27 \\
\hline 4590.974 & O II (15) & 0.350 & $\mathrm{a}$ & 0.088 & -50 & -32 & 5676.015 & N II (3) & -0.368 & $\mathrm{a}$ & 0.106 & -37 & -30 \\
\hline 4596.160 & O II (15) & 0.225 & $\mathrm{a}$ & 0.075 & -49 & & 5679.554 & N II (3) & 0.250 & $\mathrm{a}$ & 0.200 & -33 & -31 \\
\hline 4601.481 & N II (5) & -0.428 & $\mathrm{a}$ & 0.035 & -35 & & 5686.212 & N II (3) & -0.549 & $\mathrm{a}$ & 0.049 & -35 & -11 \\
\hline 4607.149 & N II (5) & -0.507 & $\mathrm{a}$ & 0.044 & -46 & & 5696.604 & Al III (2) & 0.230 & $\mathrm{a}$ & 0.168 & -46 & -34 \\
\hline 4609.473 & O II (93) & 0.729 & $\mathrm{a}$ & 0.042 & -51 & & 5710.766 & N II (3) & -0.518 & a & 0.074 & -43 & -25 \\
\hline 4613.670 & O II (92) & -1.040 & $\mathrm{a}$ & & blend & & 5722.730 & Al III (2) & -0.070 & $\mathrm{a}$ & 0.123 & -46 & -31 \\
\hline 4613.868 & N II (5) & -0.665 & $\mathrm{a}$ & & weak & & 5730.660 & N II (3) & -1.704 & $\mathrm{a}$ & 0.020 & -40 & \\
\hline 4619.249 & C II & 1.142 & $\mathrm{a}$ & 0.014 & -49 & & 5739.734 & Si III (4) & -0.160 & a & 0.220 & -47 & -34 \\
\hline 4621.241 & O II (92) & -1.150 & $\mathrm{a}$ & & blend & & 5780.410 & DIB & & $\mathrm{a}$ & 0.100 & -10 & \\
\hline 4621.396 & N II (5) & -0.514 & $\mathrm{a}$ & 0.012 & -35 & & 5797.030 & DIB & & a & 0.040 & -8 & \\
\hline 4630.543 & N II (5) & 0.094 & $\mathrm{a}$ & 0.106 & -40 & -28 & 5833.938 & Fe III (114) & 0.616 & $\mathrm{a}$ & 0.037 & -40 & \\
\hline 4638.851 & O II (1) & -0.332 & $\mathrm{a}$ & 0.107 & -50 & -27 & 5875.661 & He I (11) & 0.740 & $\mathrm{e}$ & & -39 & -22 \\
\hline 4641.810 & O II (1) & 0.054 & $\mathrm{a}$ & 0.215 & -53 & -29 & & & & $\mathrm{a}$ & & 8 & -35 \\
\hline 4643.090 & N II (5) & -0.359 & $\mathrm{a}$ & 0.052 & -40 & -28 & 5889.951 & $\mathrm{Na} I(1)$ & 0.117 & $\mathrm{a}$ & 0.160 & & -72 \\
\hline 4647.419 & C III (1) & 0.070 & $\mathrm{a}$ & 0.070 & -50 & -34 & & & & a & 0.070 & -67 & -66 \\
\hline 4649.138 & O II (1) & 0.308 & $\mathrm{a}$ & 0.292 & -47 & -30 & & & & a & 0.080 & & -52 \\
\hline 4650.838 & O II (1) & -0.361 & $\mathrm{a}$ & 0.160 & -50 & -29 & & & & $\mathrm{a}$ & 0.125 & & -28 \\
\hline 4651.018 & C III (1) & -0.432 & $\mathrm{a}$ & & blend & & & & & $\mathrm{a}$ & 0.330 & -17 & -11 \\
\hline 4661.635 & O II (1) & -0.278 & $\mathrm{a}$ & 0.135 & -47 & -30 & 5895.924 & $\mathrm{Na} I$ (1) & -0.184 & $\mathrm{a}$ & 0.125 & & -73 \\
\hline 4673.732 & O II (1) & -1.089 & a & 0.043 & -41 & & & & & a & 0.055 & -68 & -65 \\
\hline 4676.231 & O II (1) & -0.395 & $\mathrm{a}$ & 0.118 & -44 & -33 & & & & $\mathrm{a}$ & 0.060 & & -52 \\
\hline 4696.356 & O II (1) & -1.380 & $\mathrm{a}$ & 0.009 & -45 & & & & & $\mathrm{a}$ & 0.090 & & -28 \\
\hline 4699.215 & O II (25) & 0.270 & $\mathrm{a}$ & 0.047 & -54 & & & & & $\mathrm{a}$ & 0.300 & -16 & -10 \\
\hline 4703.209 & O II (40) & 0.538 & $\mathrm{a}$ & 0.013 & -64 & & 5957.559 & Si II (4) & -0.301 & $\mathrm{e}$ & 0.026 & -49 & -54 \\
\hline 4705.343 & O II (25) & 0.476 & $\mathrm{a}$ & 0.062 & -51 & & 5978.930 & Si II (4) & 0.004 & $\mathrm{e}$ & 0.090 & -44 & -53 \\
\hline 4710.012 & O II (24) & -0.226 & $\mathrm{a}$ & 0.019 & -48 & & 6195.990 & DIB & & $\mathrm{a}$ & 0.030 & -10 & \\
\hline 4713.171 & $\mathrm{He} \mathrm{I}(13)$ & -0.976 & $\mathrm{a}$ & 0.140 & -28 & -22 & 6203.060 & DIB & & $\mathrm{a}$ & 0.050 & -19 & \\
\hline 4803.287 & N II (20) & -0.113 & $\mathrm{a}$ & 0.023 & -53 & & 6347.109 & Si II (2) & 0.297 & $\mathrm{e}$ & 0.171 & -57 & \\
\hline 4813.333 & Si III (9) & 0.850 & $\mathrm{a}$ & & weak & & 6371.371 & Si II (2) & -0.003 & $\mathrm{e}$ & 0.065 & -54 & \\
\hline 4814.550 & {$[\mathrm{FeII}](20 \mathrm{~F})$} & & $\mathrm{e}$ & 0.025 & -56 & -52 & 6379.616 & N II (2) & -0.951 & $\mathrm{a}$ & 0.041 & -32 & \\
\hline 4819.688 & Si III (9) & 0.998 & $\mathrm{a}$ & 0.060 & -54 & & 6402.246 & Ne I (1) & 0.360 & $\mathrm{a}$ & 0.057 & -37 & \\
\hline 4828.957 & Si III (9) & 1.111 & a & 0.060 & -51 & & 6548.100 & {$[\mathrm{~N} \mathrm{I}](1 \mathrm{~F})$} & & $\mathrm{e}$ & 0.050 & -55 & \\
\hline 4856.594 & O II (29) & -0.583 & a & 0.060 & -47 & & 6562.797 & H I (1) & 0.710 & $\mathrm{e}$ & 13.0 & -35 & \\
\hline 4861.323 & H I (1) & -0.020 & $\mathrm{e}$ & 1.80 & -45 & -29 & 6578.053 & C II (2) & -0.026 & $\mathrm{a}$ & 0.258 & -30 & \\
\hline 4905.350 & {$[\mathrm{FeII}](20 \mathrm{~F})$} & & $\mathrm{e}$ & 0.006 & -58 & & 6582.882 & C II (2) & -0.328 & a & 0.057 & -31 & \\
\hline 4906.830 & O II (28) & -0.160 & $\mathrm{a}$ & 0.037 & -58 & & 6583.600 & {$[\mathrm{~N} \mathrm{I}](1 \mathrm{~F})$} & & $\mathrm{e}$ & & bl. & \\
\hline 4921.931 & He I (48) & -0.435 & a & 0.470 & -32 & -42 & 6605 & UN & & $\mathrm{e}$ & 0.110 & & \\
\hline 4924.525 & O II (28) & 0.074 & $\mathrm{a}$ & 0.037 & -53 & & 6613.630 & DIB & & $\mathrm{a}$ & 0.030 & -16 & \\
\hline 4994.367 & N II (24) & -0.069 & $\mathrm{a}$ & 0.016 & -50 & & 6678.154 & He I (46) & 0.329 & a & 0.258 & -15 & \\
\hline 5001.339 & N II (19) & 0.661 & $\mathrm{a}$ & 0.110 & -48 & -33 & 6721.384 & O I (4) & -0.609 & a & 0.057 & -48 & \\
\hline 5005.154 & N II (19) & 0.592 & $\mathrm{a}$ & 0.094 & -51 & -33 & 6779.939 & C II (14) & 0.024 & $\mathrm{a}$ & & bl. & \\
\hline 5007.333 & N II (24) & 0.171 & $\mathrm{a}$ & 0.042 & -48 & -30 & 6780.599 & C II (14) & -0.377 & $\mathrm{a}$ & & bl. & \\
\hline 5010.622 & N II (4) & -0.606 & $\mathrm{a}$ & 0.045 & -42 & -29 & 6783.908 & C II (14) & 0.304 & $\mathrm{a}$ & 0.054 & -43 & \\
\hline 5012.032 & N II (64) & 0.136 & a & 0.009 & -58 & & 6787.207 & C II (14) & -0.378 & a & 0.016 & weak & \\
\hline \multirow[t]{2}{*}{5015.678} & $\mathrm{He}$ I (4) & -0.820 & $\mathrm{e}$ & & -67 & -48 & 6791.466 & C II (14) & -0.271 & $\mathrm{a}$ & 0.025 & weak & \\
\hline & & & $\mathrm{a}$ & & -23 & -14 & 6798.104 & C II (14) & -1.077 & $\mathrm{a}$ & 0. & weak & \\
\hline 5041.024 & Si II (5) & 0.291 & $\mathrm{e}$ & 0.018 & -55 & -58 & 6800.683 & C II (14) & -0.345 & $\mathrm{a}$ & 0.020 & weak & \\
\hline 5044.356 & C II (35) & -0.500 & $\mathrm{a}$ & & blend & & 7065.246 & He I (10) & -0.205 & $\mathrm{e}$ & & -58 & \\
\hline 5045.103 & N II (4) & -0.407 & $\mathrm{a}$ & 0.082 & -55 & -33 & & & & $\mathrm{a}$ & & 11 & \\
\hline 5047.117 & C II (35) & -1.000 & $\mathrm{a}$ & & blend & & 7771.941 & O I (1) & 0.369 & $\mathrm{a}$ & 0.334 & -26 & \\
\hline 5047.738 & He I (47) & -1.602 & $\mathrm{a}$ & 0.110 & -34 & -28 & 7774.161 & O I (1) & 0.223 & a & 0.263 & -25 & \\
\hline 5056.017 & Si II (5) & 0.639 & $\mathrm{e}$ & 0.090 & -62 & -53 & 7775.390 & O I (1) & 0.001 & $\mathrm{a}$ & 0.150 & -28 & \\
\hline
\end{tabular}




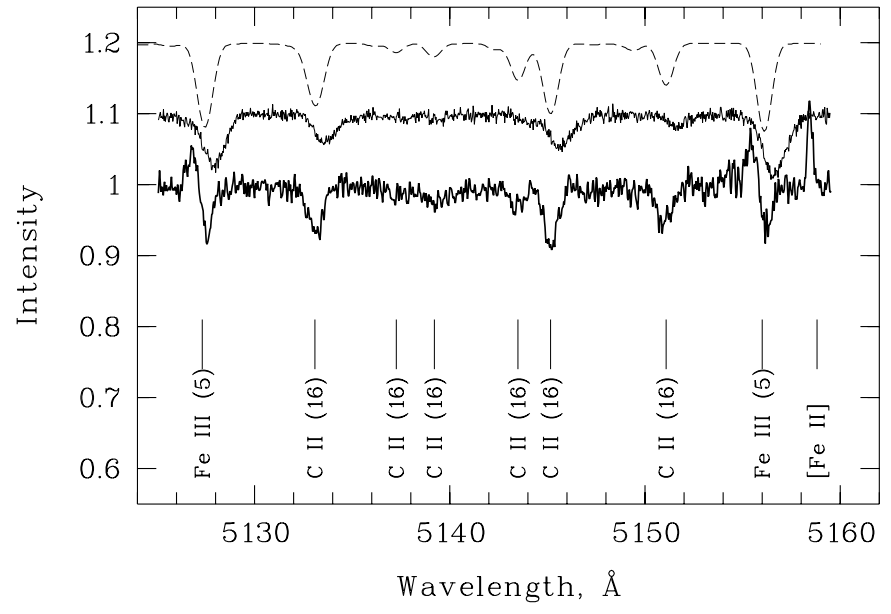

Fig. 2. Carbon lines in the spectrum of IRAS 01005+7910 (thick line). Other lines have the same meaning as in Fig. 1.

Overall, the spectrum of IRAS 01005 is very similar to that of a low-mass post-AGB star V1853 Cyg $=\mathrm{LS} \mathrm{II}+34^{\circ} 26$ (B1.5 ra, García-Lario et al. 1997; Arkhipova et al. 2001a). For example, V1853 Cyg exhibits a strong oxygen IR-triplet at $\lambda 7773 \AA\left(W_{\lambda}=0.95 \AA\right)$, whose intensity increases with luminosity (Faraggiana et al. 1988). In the spectrum of IRAS 01005 this triplet has $W_{\lambda}=0.75 \AA$, confirming its high luminosity. However, we cannot estimate the latter due to a lack of hot stars with the measured strength of the triplet.

Since the spectrum of IRAS 01005 contains many absorption lines without visible emission components (see Table 2), we can estimate abundances of several chemical elements in its atmosphere. However, we have to keep in mind that both the model parameters and chemical composition, determined for such a hot and luminous star with the unstable and extended gaseous-dusty envelope in the framework of a static planeparallel atmospheric model under the LTE approach, can be considered as a first approximation only.

All atomic parameters for the lines from Table 2 (oscillator strenghts, damping constants, etc.) were taken from the VALD database (Piskunov et al. 1995). Note that virtually all the He I lines are distorted by emission components, therefore we were not able to estimate the He content.

The chemical abundances were calculated using the Kurucz (1993) models and Kurucz's WIDTH9 program. The effective temperature $\left(T_{\text {eff }}=21500 \pm 500 \mathrm{~K}\right)$ and the surface gravity $(\log g=3.0 \pm 0.3)$ values were improved by using the criterion of the $\mathrm{Si}$ II/Si III ionization balance, i.e. equality of the Si II and $\mathrm{Si}$ III abundances. The estimated uncertainties in the abundances caused by the errors in $T_{\text {eff }}$ and $\log g$ are equal to \pm 0.2 dex, which are similar to those due to the errors of the equivalent widths. A good agreement of the abundances derived for the pair $\mathrm{C}_{\mathrm{II}} / \mathrm{C}_{\text {III }}$ supports the above model atmosphere parameters. In order to estimate the microturbulent velocity $\xi_{\mathrm{t}}$, we used the numerous well-measured $\mathrm{O}_{\text {II }}$ absorption lines and found that such a $\xi_{\mathrm{t}}$ value produced a zero slope in a diagram of $\log$ abundances calculated for individual $\mathrm{O}_{\text {II }}$ lines versus equivalent widths. Formal application of the generally adopted method leads to a very large value of $\xi_{\mathrm{t}}=45 \pm 5 \mathrm{~km} \mathrm{~s}^{-1}$. It is evident that the supersonic value of $\xi_{\mathrm{t}}$ is mainly caused by use of a plane-parallel static atmosphere model approximation for a high-luminosity and high-temperature star with an unstable atmosphere. Takeda (1992) showed that the microturbulent velocity suddenly grows when the atmosphere becomes unstable, because the radiation force is larger than the gravitational force. Such unrealistically large microturbulent velocities, obtained under the LTE-approach, is a well-known fact in hot star spectra simulations (Takeda 1977). Gies \& Lambert (1992) showed that, taking into account deviations from the LTE approximation, the microturbulent velocity can be reduced from $25-30$ to $8-11 \mathrm{~km} \mathrm{~s}^{-1}$. To reduce systematic errors and derive more realistic chemical abundances, we did not take into account all the strong lines with $W_{\lambda} \geq 80 \mathrm{~m} \AA$, since the weak lines are practically independent of the microturbulent velocity. A typical value of $\xi_{\mathrm{t}}=15 \pm 5 \mathrm{~km} \mathrm{~s}^{-1}$ for high-luminosity hot stars (Gies \& Lambert 1992; Parthsarathy et al. 2000) within the LTE-approach was adopted in our calculations.

To illustrate the role of non-LTE effects for the IRAS 01005 spectrum we calculated an oxygen abundance from the lines of both ions and neutral atoms. It is well known that the lines of the oxygen IR-triplet at $\lambda 7773 \AA$ are very sensitive to nonLTE effects. Using the equivalent widths of these lines from Table 2 we obtained $\epsilon\left(\mathrm{O}_{\mathrm{I}}\right)=9.49$, while a lower value of $\epsilon\left(\mathrm{O}_{\text {II }}\right)=8.24$ was derived from the weak $\mathrm{O}_{\text {II }}$ lines.

The average chemical abundances for different species are presented in Table 3. The abundances are given on the usual scale, $\epsilon(\mathrm{X})=n(\mathrm{X}) / n(\mathrm{H})$ with $\log \epsilon(\mathrm{H})=12$. The chemical composition for a normal supergiant 9 Cep (Gies \& Lambert 1992) is given for comparison.

As follows from Table 3, the atmospheric abundances of all the elements found for IRAS 01005 and for a normal supergiant $9 \mathrm{Cep}$ are different. The iron content $[\mathrm{Fe} / \mathrm{H}]_{\odot}=-0.31$ of IRAS 01005 is slightly smaller relative to the solar one, while it is larger for $9 \mathrm{Cep}\left([\mathrm{Fe} / \mathrm{H}]_{\odot}=+0.18\right)$. The abundances of such metals as $\mathrm{Mg}, \mathrm{Al}$, and $\mathrm{Si}$ show the same feature in both objects. As follows from the results by Gies \& Lambert (1992), a chemical abundance pattern in the case of 9 Cep agrees with the evolutionary stage of a massive supergiant: carbon is underabundant in its metal-rich atmosphere, contaminated by the $\mathrm{CN}$-cycle products. The $\mathrm{CNO}$-group behaviour in the IRAS 01005 atmosphere (the carbon abundance is enhanced relative to the solar one, $[\mathrm{C} / \mathrm{Fe}]_{\odot}=+0.08$, while the oxygen content is deficient, $[\mathrm{O} / \mathrm{Fe}]_{\odot}=-0.32$ ) is opposite to that of 9 Cep. These differences in the chemical abundance picture allow us to rule out a hypothesis that IRAS 01005 is a normal supergiant.

We note here that the main spectral peculiarities, the atmospheric parameters $T_{\text {eff }}=21500 \mathrm{~K}, \log g=3.0$, and the atmospheric abundances of IRAS 01005 are similar to those of the post-AGB object IRAS $18062+2410$, whose highresolution spectra were studied by Arkhipova et al. (2001a) and Parthasarathy et al. (2000). The most remarkable difference between IRAS 01005 and IRAS $18062+2410$ is the behaviour of the CNO abundances (Table 3). For IRAS 18062+2410, the Nand $\mathrm{O}-$ abundances are similar to the metallicity at a strong C-deficiency. For IRAS 01005 we see a different picture: the $\mathrm{C}-$ and $\mathrm{N}$-abundances follow the iron content at a large oxygen deficiency. The carbon/oxygen ratio is $\mathrm{C} / \mathrm{O}<1$ for 
Table 3. The chemical composition of IRAS 01005, $\log \epsilon(\mathrm{X})$ (for $\log \epsilon(\mathrm{H})=12.0$ ). $n$ refers to the number of lines used, and $\sigma$ is the uncertainty derived from the measurements of individual lines. Atmospheric parameters $\left(T_{\mathrm{eff}}(\mathrm{K}), \log g, \xi_{\mathrm{t}}\left(\mathrm{km} \mathrm{s}^{-1}\right)\right)$ of the stars are given below their names. The element abundances for the solar photosphere are taken from Grevesse et al. (1996).

\begin{tabular}{|c|c|c|c|c|c|c|c|c|}
\hline \multicolumn{2}{|c|}{$\overline{\text { Sun }}$} & \multirow{2}{*}{$\begin{array}{c}\text { IRAS } 18062+2410^{1} \\
22000,3.0,15 \\
\log \epsilon(\mathrm{X})\end{array}$} & \multirow[b]{2}{*}{$X$} & \multirow{2}{*}{$\begin{array}{c}9 \mathrm{Cep}^{2} \\
19040,2.61,28.9 \\
\log \epsilon(\mathrm{X})\end{array}$} & \multicolumn{4}{|c|}{$\begin{array}{c}\text { IRAS } 01005+7910 \\
21500,3.0,15\end{array}$} \\
\hline $\mathrm{X}$ & $\log \epsilon(\mathrm{X})$ & & & & $\log \epsilon(\mathrm{X})$ & $n$ & $\sigma$ & {$[\mathrm{X} / \mathrm{H}]_{\odot}$} \\
\hline \multirow[t]{2}{*}{$\bar{C}$} & \multirow[t]{2}{*}{8.55} & 6.95 & C II & \multirow[t]{2}{*}{8.12} & 8.32 & 6 & \multirow[t]{2}{*}{0.12} & -0.23 \\
\hline & & & C III & & 8.42 & 1 & & -0.13 \\
\hline $\mathrm{N}$ & 7.97 & 7.27 & N II & 8.03 & 7.70 & 10 & 0.25 & -0.27 \\
\hline $\mathrm{O}$ & 8.87 & 8.27 & O II & 8.66 & 8.24 & 12 & 0.21 & -0.63 \\
\hline $\mathrm{Mg}$ & 7.58 & 7.08 & $\mathrm{Mg}$ II & & $7.44^{3}$ & 1 & & -0.13 \\
\hline $\mathrm{Al}$ & 6.47 & 5.87 & Al III & & 5.82 & 2 & & -0.65 \\
\hline \multirow[t]{2}{*}{$\mathrm{Si}$} & \multirow[t]{2}{*}{7.55} & 6.75 & Si II & \multirow[t]{2}{*}{6.87} & 7.41 & 2 & & -0.14 \\
\hline & & & Si III & & 7.40 & 5 & 0.20 & -0.15 \\
\hline $\mathrm{Fe}$ & 7.50 & 6.90 & $\mathrm{Fe}$ III & 7.68 & 7.19 & 4 & 0.20 & -0.31 \\
\hline
\end{tabular}

${ }^{1}$ Results from Parthasarathy et al. (2000) are recalculated using the solar abundances from Col. 2.

${ }^{2}$ From Gies \& Lambert (1992).

${ }^{3}$ The Mg abundance may be overestimated since it was derived from strong $\left(W_{\lambda}=190 \mathrm{~m} \AA\right)$ line.

IRAS $18062+2410$ while for IRAS 01005 the ratio $\mathrm{C} / \mathrm{O}>1$ is in agreement with details in its IR-spectrum (Hrivnak et al. 2000, see Sect. 1). Therefore we confirmed a result by Hrivnak et al. (2000) that the central star of IRAS 01005 belongs to the group of carbon-rich PPNe.

\subsection{Spectral variability}

In the spectra obtained in 2000, the hydrogen Balmer lines $\mathrm{H} \alpha$, $\mathrm{H} \beta$ and $\mathrm{H} \gamma$ have asymmetric single-peaked emission profiles (Fig. 3) with a maximum intensity at a $V_{\mathrm{r}}$ of $-40 \mathrm{~km} \mathrm{~s}^{-1}$. The intensity of the blue wing decreases faster at low velocities than that of the red wing, while the situation is reversed at velocities more than $40 \mathrm{~km} \mathrm{~s}^{-1}$ from the line maximum. Such a profile can be a combination of two emission components with different intensities and a component due to self-absorption. In 2001 the $\mathrm{H} \alpha$ and $\mathrm{H} \beta$ profile shape became double-peaked with the intensities, which differ by an order of magnitude.

The He I line profiles vary from straight to inverse PCygtype through almost pure emission or absorption (Fig. 3). Furthermore, in the spectrum obtained on December 02, 2001 with $R=60000$ a straight PCyg-type profile of the $\lambda 5876 \AA$ line and an inverse P Cyg-type profile of the $\lambda 5016 \AA$ line are seen together. At the same time, in this spectrum the He I line at $\lambda 4922 \AA$, which does not show emission wings, is split into two absorption components, as if a weak emission in the core is present. Finally, on July 06, 2000 the $\lambda 4922 \AA$ and $\lambda 6678 \AA$ lines have inverse PCyg-type profiles with the emission and absorption components at -75 and $-27 \mathrm{~km} \mathrm{~s}^{-1}$, respectively. However, on July 13, 2000 noticeable emission components are absent in both lines.

The $V_{\mathrm{r}}$ behaviour of individual lines is more complicated. The $V_{\mathrm{r}}$ of both the absorption and emission lines correlate with the oscillator strengths (Fig. 4): the stronger lines have more negative velocities. This is more likely a result of a velocity gradient in the expanding envelope and atmosphere of the supergiant. Furthermore, the slope of the linear relationship varies from one spectrum to another (Fig. 4). The systemic velocity we define as a limiting velocity of the lines with small oscillator strengths. For all our spectra this limit is $-23 \pm 3 \mathrm{~km} \mathrm{~s}^{-1}$.

In Table 4 we show the heliocentric $V_{\mathrm{r}}$ derived from absorption and emission lines and/or their components at different epochs. A typical uncertainty of the $V_{\mathrm{r}}$ measurements for a single line is about $1 \mathrm{~km} \mathrm{~s}^{-1}$ for the spectrometer NES, $2 \mathrm{~km} \mathrm{~s}^{-1}$ for LYNX, and $4 \mathrm{~km} \mathrm{~s}^{-1}$ for PFES. A significant shift $\left(-15 \mathrm{~km} \mathrm{~s}^{-1}\right)$ in most of the absorption line $V_{\mathrm{r}}$ (except for those of Fe III) occurred in December 2001. This phenomenon may be due to changes in the star's atmospheric structure, which may also cause the disappearance of the $\mathrm{H} \beta$ line absorption component. Pulsations as a mechanism, responsible for similar $V_{\mathrm{r}}$ variations, was suggested by García-Lario et al. (1997) for V1853 Cyg. Our present time coverage does not allow us to comment on other possible reasons for it, such as orbital motion in a binary system.

In Fig. 5 the Na I resonance doublet profiles, obtained with the $R=60000$ on two different dates, are shown. We were able to distinguish five absorption components with velocities of $-11,-28,-52,-65$, and $-73 \mathrm{~km} \mathrm{~s}^{-1}$. In addition, the absorption profile of the $\lambda 5890 \AA$ line in the spectrum of December 2, 2001 is superseded on a broad high-velocity emission component, whose width is the same as those of the hydrogen line emission components. In the other spectrum there are no noticeable emission components. The first and the strongest absorption component has a $V_{\mathrm{r}}$ of $-3 \mathrm{~km} \mathrm{~s}^{-1}$ with respect to local standard of rest, and is most likely interstellar. The radio observations of the CO emission (Grenier et al. 1989) and those of the interstellar $\mathrm{Na}$ I lines for nearby stars in the direction of IRAS 01005 (e.g., Welty et al. 1994) give the LSR velocity for the interstellar medium from -8 to $+10 \mathrm{~km} \mathrm{~s}^{-1}$.

The next Na I component in the spectrum of IRAS 01005 (at $-28 \mathrm{~km} \mathrm{~s}^{-1}$ ) is located very close to the systemic velocity. It may be formed in the outer, slowest $\left(V_{\exp } \approx 5-10 \mathrm{~km} \mathrm{~s}^{-1}\right)$, and optically thin part of the circumstellar envelope. The three remaining components may arise either in more rapidly expanding shells or in the interstellar medium. 

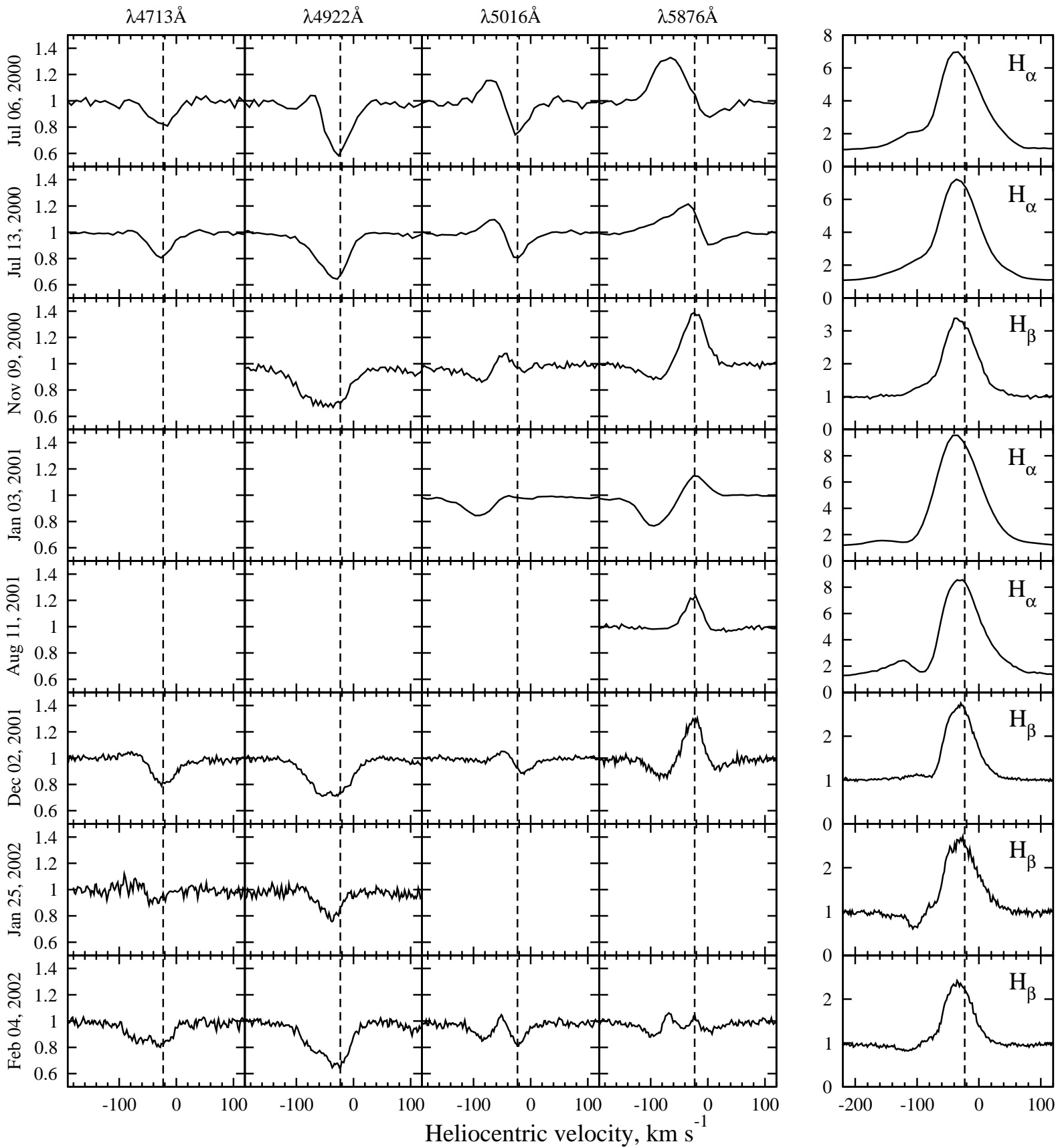

Fig. 3. The He $\mathrm{I}$ and Balmer line profile variations. The suggested systemic velocity $-23 \mathrm{~km} \mathrm{~s}^{-1}$ is shown by vertical dashed lines.

\section{Discussion}

The spectral features mentioned above led us to the following suggestions. The system of IRAS 01005 contains a highluminosity early-B type star surrounded by a gaseous envelope. The transformation of the He I line profiles from inverse (in 2000) to straight (in 2001) P Cyg-type is suggestive of a change from accretion to outflow in the inner parts of the envelope. The increasing separation between the emission components of the $\mathrm{H} \alpha$ line observed simultaneously supports the outflow hypothesis. The co-existence of the straight and inverse PCygtype profiles in different He I lines in one spectrum suggests that accretion and outflow can be present at the same time, however probably in different parts of the envelope. The difference in the profile shapes of the Balmer and He I lines may be due to a more spherical zone of the He I line formation, as it has been suggested for an LBV candidate MWC 314 (Frémat et al. 1998).

The presence of the relation between the line velocity and the oscillator strength may be due to the velocity gradient in the unstable atmosphere of IRAS 01005 . Phases of atmosphere instability from time to time alternate with phases of a relative quiescence, characterized by a low or negligible velocity gradient (Fig. 4).

Given a high luminosity, the star should be distant. From his optical photometry, $\mathrm{Hu}$ (2001) suggested that the interstellar extinction $A_{V}=1.2$, while not attempting a distance estimate, 
Table 4. Heliocentric $V_{\mathrm{r}}\left(\mathrm{km} \mathrm{s}^{-1}\right)$ in the spectrum of IRAS 01005. The number of lines used for the measurements is given in parentheses.

\begin{tabular}{|c|c|c|c|c|c|c|}
\hline 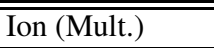 & Jul. 2000 & Nov. 2000 & "Aug. 2001 & 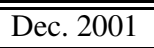 & Jan. 2002 & Feb. 2002 \\
\hline & & \multirow{2}{*}{\multicolumn{2}{|c|}{ Absorption lines }} & & & \\
\hline C II (16) & $-49(4)$ & & & $-31(3)$ & & $-50(3)$ \\
\hline N II (3) & $-38(6)$ & $-50(5)$ & $-41(5)$ & $-25(5)$ & & $-43(5)$ \\
\hline O II (1) & $-47(8)$ & & & $-30(6)$ & $-41(7)$ & $-47(7)$ \\
\hline Al III (2) & $-46(2)$ & $-51(2)$ & $-46(2)$ & $-33(2)$ & & $-47(2)$ \\
\hline Si III $(2,4)$ & $-50(4)$ & $-48(1)$ & $-45(1)$ & $-32(2)$ & $-39(3)$ & $-43(1)$ \\
\hline Fe III (5) & $-27(2)$ & & & $-23(2)$ & & $-23(1)$ \\
\hline $\mathrm{H} \alpha$ & & & -92 & & & \\
\hline $\mathrm{H} \beta$ & & & & -84 & $-105,-75$ & $-118,-80$ \\
\hline $\mathrm{H} \gamma$ & -179 & & & & $-103,-73$ & \\
\hline$[\mathrm{FeII}](7,18-20)$ & $-58(6)$ & & iission lines & $-51(3)$ & $-51(1)$ & $-53(3)$ \\
\hline Fe III (5) & $-72(2)$ & & & $-67(2)$ & & \\
\hline Si II $(2,4,5)$ & $-54(6)$ & $-49(3)$ & $-55(4)$ & $-56(4)$ & & $-50(4)$ \\
\hline $\mathrm{H} \alpha$ & -35 & & $-32,-124$ & & & \\
\hline $\mathrm{H} \beta$ & -45 & -34 & & $-100,-30$ & -32 & -35 \\
\hline $\mathrm{H} \gamma$ & -43 & & & & -39 & \\
\hline
\end{tabular}

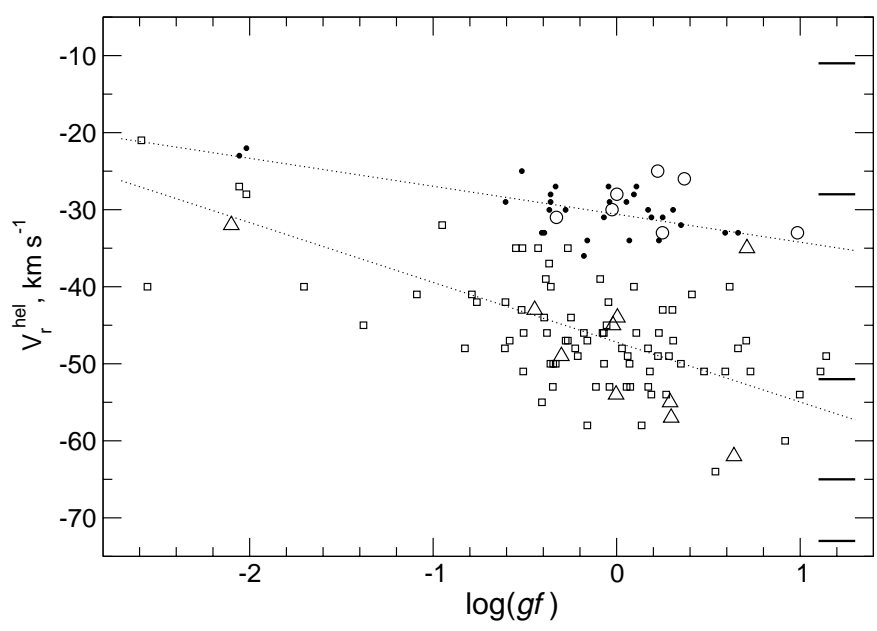

Fig. 4. Relations between the heliocentric $V_{\mathrm{r}}$ of the absorption lines and oscillator strengths at two epochs July 13, 2000 (open squares) and December 02, 2001 (dots) (see Table 2). The dotted lines show the linear fits to the relations. The emission lines in the July 2000 spectrum (open triangles) show the same distribution as the absorption lines. The open circles denote the group of the strong absorption lines in the July 2000 spectrum (O I triplet $\lambda 7773 \AA$, Mg II $\lambda 4481 \AA$, C II $\lambda \lambda 6578,6583 \AA$ and N II $\lambda 5680 \AA$ ). The velocities of the $\mathrm{NaI} \mathrm{D}_{1,2}$ line componets are shown by thick horisontal lines.

as IRAS 01005 is peculiar. Indeed, both luminosity and kinematic distance calibrations are mostly based on the galactic disk stars, while IRAS 01005 most likely belongs to the halo. Our result for $A_{V}$ (Sect. 3.1) might imply that part of the extinction is circumstellar.

Let us try to estimate the luminosity and distance of IRAS 01005 by using the values $T_{\text {eff }}=21500 \mathrm{~K}$ and $\log g=3.0$ we obtained. As follows from the locus of likely post-AGB stars in the $\log g$ vs. $T_{\text {eff }}$ plane (Fig. 3 from Schönberner \& Blöcker 1993), we can adopt a mass of the object of $M \approx 0.57 \pm 0.05 M_{\odot}$. According to theoretical postAGB evolutionary tracks (Blöcker 1995), such an object would

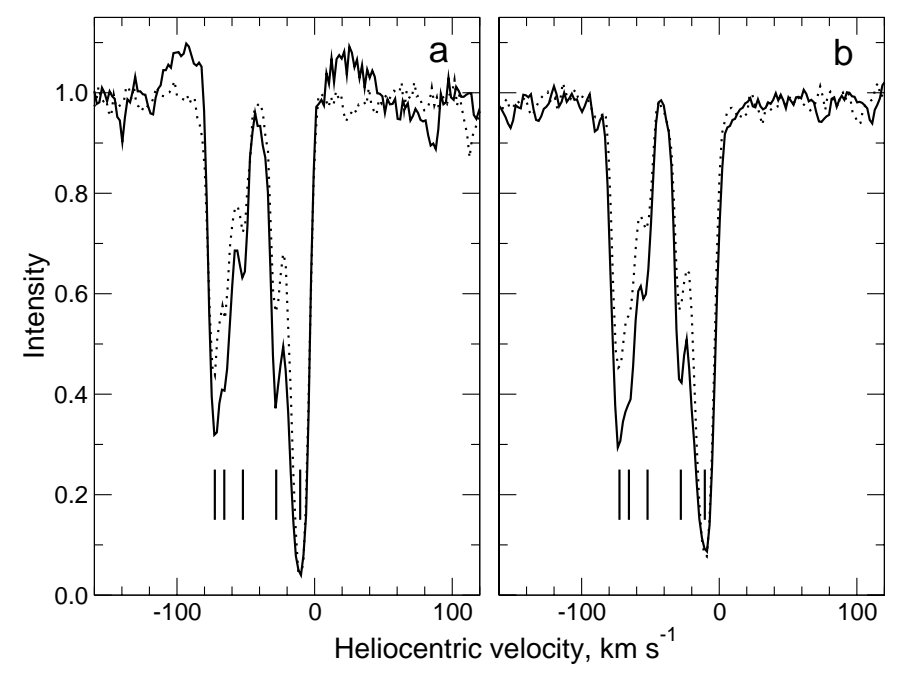

Fig. 5. The $\mathrm{Na}$ I $\mathrm{D}_{1}$ (dotted) and $\mathrm{D}_{2}$ (solid) line profiles in high resolution spectra of Dec. 2001 (a) and Feb. 2002 (b).

have a luminosity of $\log \left(L / L_{\odot}\right)=3.6\left(M_{\mathrm{bol}}=-4.3\right)$. From the observed $V=11.2 \mathrm{mag}$ (Hu 2001) and the interstellar (and circumstellar) extinction $A_{V}=1.2 \mathrm{mag}$, we have the dereddened $V_{0}=10 \mathrm{mag}$ and, accepting the bolometric correction $B C=-2 \mathrm{mag}$, we obtain $V_{0}-M_{V}=12.3 \mathrm{mag}$, that corresponds to a distance of $\sim 3 \mathrm{kpc}$. Taking into account the uncertainities in the effective temperature and surface gravity (see Sect. 3.2), we estimate an error of the distance determination to be about $20 \%$. Such a distance is consistent with the presence of the sodium D-lines absorption components at $V_{\mathrm{r}}=-52 \mathrm{~km} \mathrm{~s}^{-1}$ that may be due to the interstellar material within the Perseus arm (Münch 1957). The interstellar origin of the higher-velocity components (see Fig. 5) requires a larger distance toward the object, which would make it more luminous and more rapidly evolving along the post-AGB track. Since there is no evidence of rapid evolution, one can suggest that these components were formed in the circumstellar envelope ejected during the AGBphase. 
The reduced metallicity of IRAS $01005[\mathrm{Fe} / \mathrm{H}]_{\odot}=-0.31$ in combination with the altered $\mathrm{CNO}$-abundances $(\mathrm{C} / \mathrm{O}>1)$ and the high galactic latitude, suggests that it is a hot postAGB star. If its luminosity and, hence, mass are large enough, the star's $T_{\text {eff }}$ will increase quickly (Blöcker 1995). As a result, one can expect an increase of the emission-line strengths in the object's spectrum, because in comparison with other early-type PPNe (OY Gem and IRAS 18062+2410), IRAS 01005 shows a relatively weak emission-line spectrum. Alternatively, the latter might imply that its gaseous envelope is compact, being limited by the presence of a companion star. Another indication for binarity can be the envelope's asphericity (inferred from the emission line profiles). Therefore, further spectroscopic monitoring of IRAS 01005 is highly desirable to further constrain its properties.

\section{Conclusions}

We accomplished the first detailed indentification of the observed features in the high-resolution optical spectrum of an optical counterpart of the PPN candidate IRAS 01005. The absorption lines of $\mathrm{C}_{\mathrm{II}} / \mathrm{III}, \mathrm{N}_{\text {III }}, \mathrm{O}_{\text {III }}, \mathrm{Al}_{\mathrm{III}}, \mathrm{Si}_{\mathrm{II} / \mathrm{III}}$, and the $\mathrm{Mg}_{\text {II }} \lambda 4481 \AA$ line, as well as emission lines of $\mathrm{Si}$ II and [Fe II] were found in the spectrum. Both emission and absorption components are present in the hydrogen Balmer lines, in the resonance Na I $\mathrm{D}_{1,2}$ lines, and in the He I and Fe III lines. A significant variability of the optical spectrum was detected: the $\mathrm{He}$ I lines change their profiles from straight to inverse P Cygtype on a timescale of days-months.

A high effective temperature $T_{\text {eff }}=21500 \mathrm{~K}$, a low surface gravity $\log g=3.0$, and a reduced metallicity of IRAS 01005 $\left([\mathrm{Fe} / \mathrm{H}]_{\odot}=-0.31\right)$ in combination with the altered atmospheric $\mathrm{CNO}-$ abundances $(\mathrm{C} / \mathrm{O}>1)$ and a high galactic latitude, confirm the evolutionary state of a hot post-AGB C-rich star.

We suggest that IRAS 01005 is a high-luminosity $\log \left(L / L_{\odot}\right)=3.6$ early-B type post-AGB star at a distance of $\sim 3 \mathrm{kpc}$, surrounded by a non-spherical gaseous envelope with both observable accretion and outflow. The envelope geometry and weakness of the emission-line spectrum may indicate binarity of the system.

Acknowledgements. We are much indebted to the anonymous referee, who stimulated the analysis of the chemical composition of IRAS 01005. This work was supported in part by Russian Foundation for Basic Research (project No.02-02-16085). The research described in this publication was made possible in part by Award No. RP1-2264 of the U.S. Civilian Research \& Development Foundation for the Independent States of the Former Soviet Union (CRDF). This research has made use of the SIMBAD database operated at CDS (Strasbourg, France) and of the VALD database operated at Vienna.

\section{References}

Arkhipova, V. P., Klochkova, V. G., \& Sokol, G. V. 2001, Astron. Lett., 27,99

Arkhipova, V. P., Ikonnikova, N. P., Noskova, R. I., et al. 2001, Astron. Lett., 27, 719

Blöcker, T. 1995, A\&A, 299, 755

Decin, L., Van Winckel, H., Waelkens, C., \& Bakker, E. J. 1998, A\&A, 332, 928

Didelon, P. 1982, A\&AS, 50, 199

Faraggiana, R., Gerbaldi, M., van't Veer, C., \& Floquet, M. 1988, A\&A, 201, 259

Frémat, Y., Miroshnichenko, A. S., \& Houziaux, L. 1998, in B[e] stars, ed. A.-M. Hubert, \& C. Jaschek (Kluwer Acad. Publ.), 97

Galazutdinov, G. A. 1992, Preprint of the Spec. Astrophys. Obs., No. 92

García-Lario, P., Parthasarathy, M., de Martino, D., et al. 1997, A\&A, 326,1103

Gies, D. R., \& Lambert, L. 1992, ApJ, 387, 673

Grenier, I. A., Lebrun, F., Arnaud, M., Dame, T. M., \& Thaddeus, P. 1989, ApJ, 347, 231

Grevesse, N., Noels, A., \& Sauval, A. J. 1996, ASP Conf. Ser., 99, 117

Herbig, G. H. 1993, ApJ, 407, 142

Hrivnak, B., Volk, K., \& Kwok, S. 2000, ApJ, 535, 275

Hu, J. Y. 2001, in Post-AGB Objects as a Phase of Stellar Evolution, Proc. of the Torun Workshop, ed. R. Szczerba, \& S. K. Górny (Kluwer Acad. Publ.), 317

Kilian, J., \& Nissen, P. E. 1989, A\&AS, 80, 255

Klochkova, V. G. 1998, Bull. Spec. Astrophys. Obs., 44, 5

Kurucz, R. L. 1993, Smithsonian Astron. Obs., CD ROM, No. 19

Lambert, D. L., Hinkle, K. H., \& Luck, R. E. 1988, ApJ, 333, 917

Lewis, B. M. 1989, ApJ, 338, 234

Likkel, L. 1989, ApJ, 344, 350

Likkel, L., Forveille, T., Omont, A., \& Morris, M. 1991, A\&A, 246, 153

Miroshnichenko, A. S., Fremat, I., Houziaux, L., et al. 1998, A\&AS, 131,469

Moore, C. E. 1945, A multiplet table of astrophysical interest

Münch, G. 1957, ApJ, 125, 42

Omont, A., Loup, C., Forveille, T., et al. 1993, A\&A, 267, 515

Panchuk, V. E., Najdenov, I. D., Klochkova, V. G., et al. 1998, Bull. Spec. Astrophys. Obs., 44, 127

Panchuk, V. E., Klochkova, V. G., Najdenov, I. D., et al. 1999a, Preprint of the Spec. Astrophys. Obs., No. 139

Panchuk, V. E., Klochkova, V. G., \& Najdenov, I. D. 1999b, Preprint of the Spec. Astrophys. Obs., No. 135

Parthasarathy, M., \& Pottasch, S. R. 1989, A\&A, 225, 521

Parthasarathy, M., García-Lario, P., Sivarani, T., Manchado, A., \& Sanz Fernandez de Cordoba, L. 2000, A\&A, 357, 241

Piskunov, N. E., Kupka, F., \& Ryabchikova, T. A. 1995, A\&AS, 112, 525

Schönberner, D., \& Blöcker, T. 1993, ASP Conf. Ser., 45, 337

Takeda, M. 1977, PASJ, 29, 439

Takeda, M. 1992, PASJ, 44, 309

Van der Veen, V. E. C. J., \& Habing, H. J. 1988, A\&A, 194, 125

Van Winckel, H. 2001, Ap\&SS, 275, 159

Van Winckel, H., Waelkens, C., \& Waters, L. B. F. M. 1995, A\&A, 239, L25

Welty, D. E., Hobbs, L. M., \& Kulkarni, V. P. 1994, A\&AS, 100, 107 